the slightest provocation should the anterior chamiber be lost. It is taut, thus allowing any escaping aqueous to run over its surface.

\title{
SEROUS CYST OF THE ORBIT CAUSEDD BY AN ANILINE PENCIL
}

BY

Frank Juler

LONDON

ON account of its rarity, the following case is thought worthy of record.

John A, aged 4 years, was admitted to Moorfields Hospital in January, 1921, with the history that he had fallen down three weeks previously and broken a pencil into his eye. The resulting swelling subsided but returned eleven days after'the injury.

On admission there was a small colourless healed wound in the centre of the upper lid of the left eye. The upper lid was swollen, and beneath it was palpable a fluctuating, fairly tense and welldefined swelling. It was non-pulsating, and gave no impulse on coughing. Definite proptosis was present, with pushing downwards of the globe, and there was limitation of movement in upward regard.

Whilst under observation the swelling increased in size.

The cyst was incised and drained. It was found to be filled with violet coloured fluid and to contain several fragments of aniline pencil, whilst the lining tissues were deeply stained.

The sinus continued to discharge for five or six weeks, and then healed, leaving some ptosis. The child was brought for refractive error three years later (March, 1924) when the movements of the lid and eyeball were found to be normal, and there was no proptosis.

Remarks. I have been unable to find any record in the literature of an orbital cyst produced by a chemical irritant. Cases are, of course, occasionally met with in which the conjunctiva and cornea are deeply stained by the aniline dye; these require careful removal of the pencil particles, or a severe caustic burn is likely to result. In the last edition of Duane's translation of Fuchs' Textbook, it is recommended to instil at once a 5 to 10 per cent. solution of tannin (Vogt). I have no experience of this treatment, but a recent case in my clinic was treated with a weak solution of methylated spirit, and no coloration remained the next day, when the eye was found to show little reaction. 